\title{
Quadcopter sensing system for risky area
}

\begin{abstract}
There are certain areas that have risks that require doing some measurements. A typical area is sanitary landfill site where there is need to monitor gas concentration periodically even after it has been closed for many years. In this paper, we will develop a sensing system having the ability to measure the gas concentration in a landfill site, and we will focus on the type of gas distribution with respect to the gas emission source. Also, we will try to present a method to prove that our sensing system will be reliable.
\end{abstract}

Keyword: Sensing; Gas distribution; Landfill; Methane 